\title{
Precisão do modelo digital de elevação (SRTM-Topodata) com base em dados de projetos de aproveitamentos hidrelétricos
}

\author{
Marcel Medinas de CAMPOS ${ }^{1 *}$, Rafael Pedrollo de PAES ${ }^{2}$, \\ Ana Rubia de Carvalho Bonilha SILVA ${ }^{2}$, Ibraim FANTIN-CRUZ ${ }^{1,2}$ \\ 'Programa de Pós-Graduação em Recursos Hídricos, Universidade Federal de Mato Grosso, Cuiabá, MT, Brasil. \\ ${ }^{2}$ Departamento de Engenharia Sanitária e ambiental, UFMT, Cuiabá, MT, Brasil. \\ *E-mail: medinasmarcel@gmail.com
}

Recebido em março/2018; Aceito em outubro/2018.

\begin{abstract}
RESUMO: A precisão altimétrica do Modelo Digital de Elevação - MDE tem sido tema de diversos estudos. Essa precisão exerce forte influência sobre as informações extraídas desses dados. Nesse contexto, o presente estudo compara dados observados em projetos de aproveitamentos hidrelétricos com dados extraídos do MDE. A comparação de dados altimétricos de informações extraídas pelo MDE com as contidas no Projeto Básico Ambiental - PBA desses empreendimentos, assumido como informação verdadeira, foi feita com o intuito de analisar o erro das informações extraídas do MDE em relação aos dados contidos no PBA e assim verificar a confiança nesse tipo de estimativa. Foram calculados o erro e o coeficiente de determinação de Pearson entre a altura da barragem (determinada com base no $\mathrm{MDE}$ ) em relação à altura apresentada no PBA. Também foi comparada uma seção topobatimétrica do PBA com a mesma seção extraída pelo MDE. O erro relativo médio e o coeficiente de determinação entre as cinco alturas (estimadas e de projeto) foi de $11 \%$ e 0,874 , respectivamente. O coeficiente de determinação, o erro médio quadrático e o erro médio entre as seções foram de 0,98, 1,56 e 0,02 , respectivamente. A análise evidenciou que há erros em relação às informações extraídas do MDE. Entretanto, considerando a escala utilizada nesse estudo, os erros foram menores que os observados na literatura. Palavras-chave: MDE validação, Altura de barragem, Seção topobatimétrica.
\end{abstract}

\section{Altimetry accuracy of the digital elevation model (SRTM-Topodata) based on designs of hydropower plants data}

\begin{abstract}
The altimetry accuracy measurements of the Digital Elevation Model - DEM have been the subject of several studies. This accuracy plays an important role on information extracted from these data. In this context, this study compares data observed in designs of hydroelectric power plants with data extracted from the DEM. The comparison between the altimetry of data extracted from the DEM with those contained in the Basic Environmental Project - BEP of these facilities, assumed as the true information, was done with the purpose of analyzing the error of the information extracted from the DEM in relation to the data contained in the BEP and thus verifying the confidence in this type of estimate. The error and the coefficient of determination between the dam height (determined based on the DEM) and the dam height presented in the BEP were calculated. Moreover, a river cross section published in the BEP was contrasted with the same cross section extracted from the DEM. The mean relative error and the coefficient of determination between the five heights (estimated and projected) was $11 \%$ and 0.874 , respectively. The coefficient of determination, mean square error and mean error between sections were $0.98,1.56$ and $-0,02$, respectively. The analysis evidenced that there are errors in relation to the information extracted from the DEM. However, considering the scale used in this study, the errors were smaller than those observed in the literature.
\end{abstract}

Keywords: DEM validation, dam height, cross section.

\section{INTRODUÇÃO}

O processamento computadorizado possibilita, cada vez mais, a análise de informações complexas em numerosos processos interativos. O geoprocessamento, por exemplo, pode ser considerado como uma das áreas da ciência que foi aprimorada nesse contexto. No entanto, os avanços tecnológicos juntamente às bases de dados não estão isentos de erros, e devem ser considerados no processamento.

O Modelo Digital de Elevação - MDE - é uma das bases de dados utilizadas no Sistema de Informações Geográficas SIG. O MDE é fundamental para os métodos de análise do terreno (LI et al., 2017) e a sua precisão é crucial (ERSKINE et al., 2007). Essa base de dados, da qual se extrai informações, deve apresentar resolução (REDDY; REDDY, 2015) e precisão, adequadas para se extrair informações precisas sobre determinado aspecto.

A gama de informações extraídas do MDE abrange diversas áreas como, por exemplo, o estudo desenvolvido por Zhou; Xiong (2018), que determina relação altimétrica do terreno e aspectos socioeconômicos, ou em previsões derivadas de modelos hidráulicos com base em MDE (LÓPEZ-VICENTE; ALVAREZ, 2018; TAN et al., 2015).

Estudos sobre a precisão altimétrica do MDE e sua implicação sobre aspectos da natureza, mostram a 
preocupação que se tem com as informações inferidas nessa base de dados como, por exemplo: Sousa et al. (2017); Walker et al. (1999); Dixon et al. (2009); Ghumman et al. (2017); Habtezion et al. (2016); Reddy; Reddy (2015).

O senso comum existente nesses estudos é a relação do erro vertical do MDE com a escala considerada nas análises, podendo resultar em erros consideráveis dependendo da fonte dos dados e da escala utilizadas nas análises. Por isso a importância da comparação e validação das informações extraídas do MDE com outras fontes de dados.

Esse estudo tem por objetivo comparar dados observados em projetos, de aproveitamentos hidrelétricos (AH), submetidos a órgãos de licenciamento com os dados extraídos do MDE. Essa extração contempla uma técnica simplificada de determinação de altura de barragem com base no MDE e imagem de satélite, e que pode auxiliar de forma pratica a obtenção dessa informação.

O MDE disponibilizado gratuitamente pelo TOPODATA abrange o território brasileiro, apresenta resolução de 30 metros, e é de fácil acesso ${ }^{1}$. Essas características propiciam o emprego dessa base de dados por diversos profissionais, e isso desencadeou o maior interesse em analisar os erros dessa base de dados.

Considerando ainda que a verificação desse MDE com base em informações de empreendimentos hidrelétricos (consequentemente dados próximos a talvegues) aumenta a relevância deste estudo frente a características extraídas em cursos d'água como, por exemplo, identificação de barreiras naturais: para inventários de potenciais hidrelétricos (LARENTIS et al., 2010) ou para gestão e mapeamento de rotas de peixes.

\section{MATERIAL E MÉTODOS}

\section{1. Área de estudo}

A área estudo compreende uma secção do represamento gerado pela hidrelétrica Pampeana Localizada no rio Juba entre os municípios de Tangará da serra e Barra do Bugres (Figura 1).

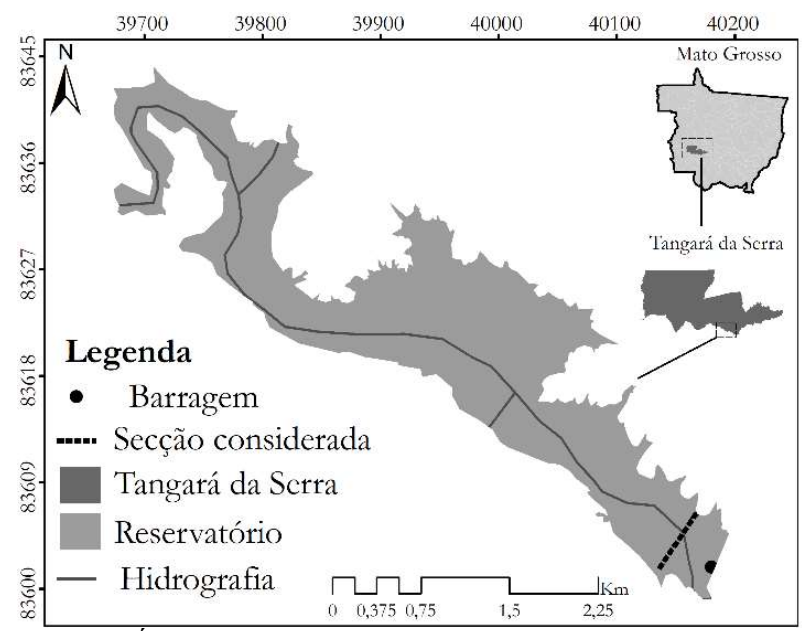

Figura 1. Área de estudo.

Figure 1. Study area.

A comparação de dados altimétricos entre aproveitamentos hidrelétricos (cujas informações estão contidas em Projeto Básico Ambiental - PBA) e as informações extraídas do MDE (Figura 2), aos mesmos aspectos, foi feita com o intuito de determinar o erro da estimativa deste último em relação aos dados contido no PBA.

O MDE empregado na análise é disponibilizado na plataforma TOPODATA (gerenciado pelo Instituto de Pesquisas Espaciais - INPE) ora gerados a partir do Shuttle Radar Topography Mission (SRTM) que forneceu, pela primeira vez na história, DEM com cobertura global de alta qualidade com níveis de resolução de aproximadamente 30 metros e 90 metros (RABUS et al., 2003).

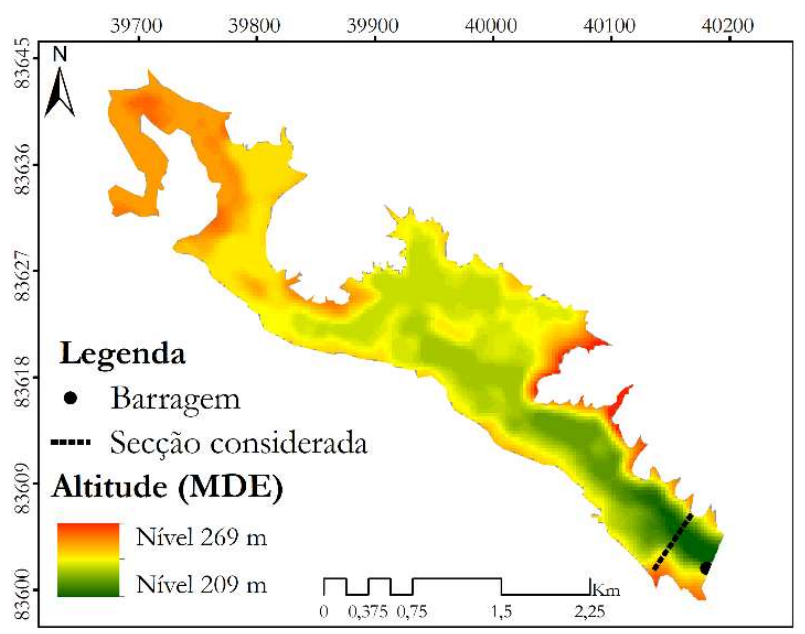

Figura 2. Modelo Digital de Elevação (MDE) utilizado no estudo. Figure 2. Digital Elevation Model (DEM) used in the study.

O Departamento de Empreendimentos Energéticos (DEE), da Secretaria Estadual do Meio Ambiente SEMA-MT, fica responsável pelos processos ambientais dos empreendimentos energéticos que inclui os $\mathrm{AH}$ que estão sob jurisdição do estado de Mato Grosso. Este departamento mantém arquivados todos os processos de licenciamento ambiental das hidrelétricas. Foi autorizada por esse departamento a pesquisa in loco dos processos de licenciamento ambiental, os quais contêm, entre outras informações, os dados de projeto dos empreendimentos.

Foram extraídas informações do PBA de cinco empreendimentos, como a altura de barragem e dados topobatimétricos do reservatório, sendo essas informações utilizadas na análise comparativa em relação a dados extraídos do MDE. Os empreendimentos, em operação na bacia do rio Paraguai, são: UHE Juba I, PCH Pampeana, PCH Ombreiras, PCH Indiavaí e PCH Salto. A comparação foi feita em duas categorias, uma que confere à altura de barramento e outro para uma dada seção topobatimétrica.

\subsection{Altura de barragem}

Para estimar a altura da barragem, foi necessário identificar, por meio de imagem de satélite, a barragem e o ponto na margem onde finda o reservatório. A cota do terreno (MDE) nesses dois pontos foi coletada, conforme a Figura 3.

A diferença entre as cotas dos pontos corresponde à altura estimada da barragem, conforme Equação 1.

$$
\mathrm{He}=\mathrm{Cr}-\mathrm{Cb}
$$

(Equação 1)

em que: $\mathrm{H}_{\mathrm{e}}=$ Altura estimada da barragem, em metros; $\mathrm{Cb}=$ Cota do terreno na barragem, em metros; $\mathrm{Cr}=\mathrm{Cota}$ na extremidade do reservatório, em metros.

\footnotetext{
${ }^{1} \mathrm{http}: / /$ www.webmapit.com.br/inpe/topodata/
} 


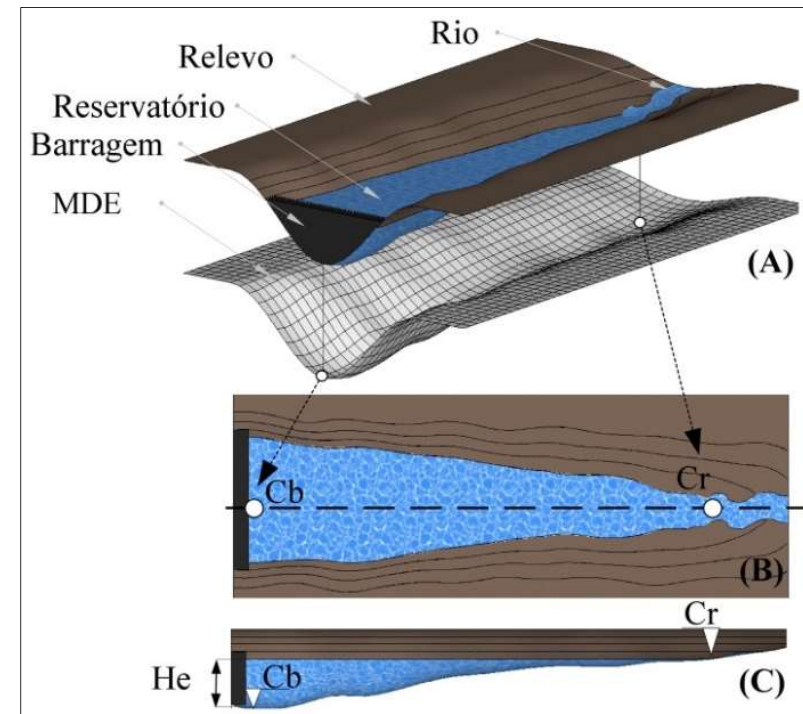

Figura 3. Método para estimar a altura da barragem: (A) Representação da imagem de satélite e MDE; (B) Cotas identificadas no MDE com auxílio da imagem; (C) Corte longitudinal com as cotas consideradas na estimativa.

Figure 1. Method to estimate the dam height: (A) Representation of the satellite image and Digital Elevation Model - DEM; (B) Elevations identified in the DEM with the aid of the image; (C) Longitudinal section with the dimensions considered in the estimation.

\subsection{Seção topobatimétrica}

Apenas uma seção topobatimétrica no projeto da $\mathrm{PCH}$ Pampeana estava disposta de maneira que possibilitou a reprodução e posterior comparação. A comparação foi feita por meio do coeficiente de correlação entre as seções topobatimétricas, do projeto e a extraída do MDE.

A seção topobatimétrica anexada ao processo de licenciamento da PCH Pampeana correspondia a uma seção transversal do rio Juba, à montante da barragem, apresentava extensão de 590 metros, com pontos de controle espaçados em 10 metros. Ela foi digitalizada para planilha eletrônica, e com base nas coordenadas dessa seção foi traçada a seção em ambiente SIG. Os valores altimétricos da topobatimetria, outrora extraídos do MDE, foram também exportados para planilha eletrônica.

O coeficiente de correlação é determinado comparando pares de dados, foi então necessário estabelecer pontos de controle de 10 metros (observada na seção do projeto) para a seção extraída do MDE. Determinou-se a função polinomial que descreveu o comportamento da cota (extraída do MDE) em relação à distância, para então determinar o coeficiente de correlação entre as seções: de projeto e extraída do MDE.

\subsection{Análise estatística}

Para comparar a altura estimada à altura contida no PBA foram determinados os erros absoluto e relativo. $\mathrm{O}$ erro absoluto, que mede a diferença absoluta entre esses valores, determinado pela Equação 2. O erro relativo, recomendado por Lin et al. (2013) para validar dados derivados de MDE, é o quociente entre o valor estimado e de projeto, definido pela Equação 3.

$$
\begin{aligned}
& E_{A}=H_{e}-H_{p} \\
& E_{R}=\frac{\left|E_{A}\right|}{H_{p}} \times 100
\end{aligned}
$$

em que: $\mathrm{E}_{\mathrm{A}}=$ Erro absoluto, em metros; $\mathrm{H}_{\mathrm{p}}=$ Altura do barramento contida no projeto, em metros; $\mathrm{H}_{\mathrm{e}}=$ Altura do barramento estimada, em metros; $\mathrm{E}_{\mathrm{R}}=$ Erro relativo, em \%.

Para análise estatística tanto das alturas estimadas quanto para a seção topobatimétrica foi empregado o coeficiente de determinação de Pearson, definido pela (Equação 4), sendo ainda determinado erro quadrático médio (Equação 5) e o erro médio (Equação 6) entre as seções, conforme GongaSaholiariliva et al. (2011).

$$
\begin{aligned}
& \mathrm{R}^{2}=\frac{\sum_{\mathrm{i}=1}^{\mathrm{n}}\left(\mathrm{P}_{\mathrm{ref}}-\overline{\mathrm{P}}_{\mathrm{ref}}\right)\left(\mathrm{P}_{\mathrm{x}}-\overline{\mathrm{P}}_{\mathrm{x}}\right)}{\sqrt{\sum_{\mathrm{i}=1}^{\mathrm{n}}\left(\mathrm{P}_{\mathrm{ref}}-\overline{\mathrm{P}}_{\mathrm{ref}}\right)^{2}} \cdot \sqrt{\sum_{\mathrm{i}=1}^{\mathrm{n}}\left(\mathrm{P}_{\mathrm{x}}-\overline{\mathrm{P}}_{\mathrm{x}}\right)^{2}}} \\
& \mathrm{EMQ}=\left\{\frac{1}{\mathrm{n}} \sum_{\mathrm{x}=1}^{\mathrm{n}} \mathrm{P}_{\mathrm{x}}-\mathrm{P}_{\mathrm{ref}}\right\}^{0,5} \\
& \mathrm{EM}=\frac{1}{\mathrm{n}} \sum_{\mathrm{x}=1}^{\mathrm{n}} \mathrm{P}_{\mathrm{x}}-\mathrm{P}_{\text {ref }}
\end{aligned}
$$

em que: $P_{x}=$ valor extraído do MDE, em metros; $P_{\text {ref }}=$ é o valor de referência (observado no Projeto Básico Ambiental do AH), em metros; e $\mathrm{n}=$ número de amostras observado no PBA, unidade.

\section{RESULTADOS}

A série temporal com as medidas de temperatura é apresentada na Figura 3 e as respectivas regressões entre os valores medidos de temperatura para os abrigos termohigrômetros projetados e o de referência na Figura 4.

As alturas de barragem contidos no PBA e estimados com base no MDE, assim como as análises estatísticas entre elas, estão dispostos na Tabela 1. Os maiores erros, tanto absoluto quanto relativo, foram constatados nas $\mathrm{PCHs}$ de Indiavaí e Salto, sendo que o erro relativo nelas foi de $13,04 \%$ e $28,82 \%$, respectivamente. $\mathrm{O}$ erro relativo médio e coeficiente de correlação entre as cinco alturas (estimadas e de projeto) foi de $11 \%$, de 0,874 (máxima correlação é 1), respectivamente.

Tabela 1. Análise comparativa entre alturas da barragem, estimada com base no MDE, e constatada em projeto.

Table 1. Comparative analysis between dam heights, estimated based on the DEM (Digital Elevation Model), and verified in design.

\begin{tabular}{lccccc}
\hline Nome & $\mathrm{H}_{\mathrm{e}}(\mathrm{m})$ & $\mathrm{H}_{\mathrm{p}}(\mathrm{m})$ & $\mathrm{E}_{\mathrm{a}}(\mathrm{m})$ & $\mathrm{E}_{\mathrm{R}}(\mathrm{m})$ & $\mathrm{R}^{2}$ \\
\hline Juba I & 18,00 & 17,50 & 0,50 & 2,86 & 0,874 \\
Pampeana & 25,00 & 26,70 & 1,70 & 6,37 & \\
Ombreiras & 11,00 & 12,00 & 1,00 & 8,33 & \\
Indiavaí & 20,00 & 23,00 & 3,00 & 13,04 & \\
Salto & 12,00 & 17,00 & 5,00 & 29,41 & \\
\hline
\end{tabular}

$\mathrm{H}_{\mathrm{e}}$ (altura estimada); $\mathrm{H}_{\mathrm{p}}$ (altura de projeto); $\mathrm{E}_{\mathrm{a}}$ (erro absoluto); $\mathrm{E}_{\mathrm{R}}$ (erro relativo); $\mathrm{R}^{2}$ (coeficiente de correlação).

A seção topobatimétrica extraída do MDE continha 19 vértices espaçados entre si em 30 metros. A regressão polinomial dessa seção (Figura 4) está representada pela linha tracejada, com coeficiente de correlação de 0,998.

A seção topobatimétrica do projeto e a função polinomial, que, em tese, representa a seção extraída do MDE, estão dispostas na Figura 5. A correlação entre os 59 pontos de controle (espaçados em 10 metros) das secções: de projeto e extraídas do MDE, foi de 0,985.

A representação dos pontos das duas secções em gráfico de dispersão e análises estatísticas estão dispostas na Figura 6, sendo que o coeficiente de correlação, erro médio quadrático, e o erro médio entre as seções foram de $0,98,1,56,-0,02$, respectivamente. 


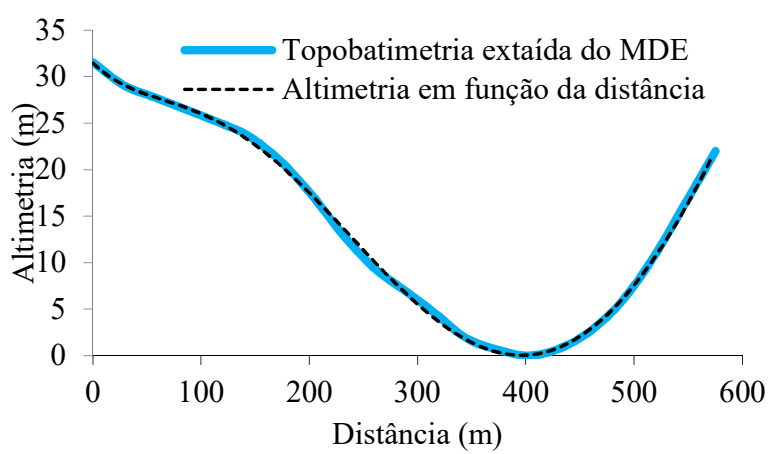

Figura 4. Regressão da seção topobatimétrica extraída do MDE. Figure 4. Regression of cross section extracted from the DEM.

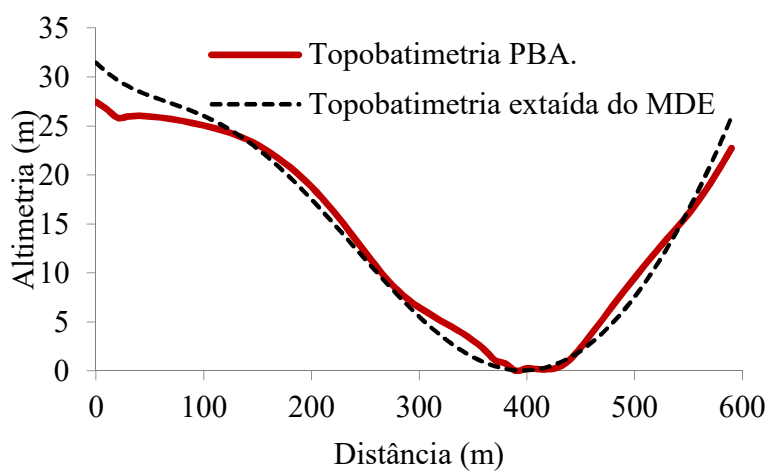

Figura 5. Comparação entre a seção topobatimétrica constatada no PBA e extraída do MDE.

Figure 2. Comparison between the cross section verified in the Basic Environmental Project and extracted from the DEM.

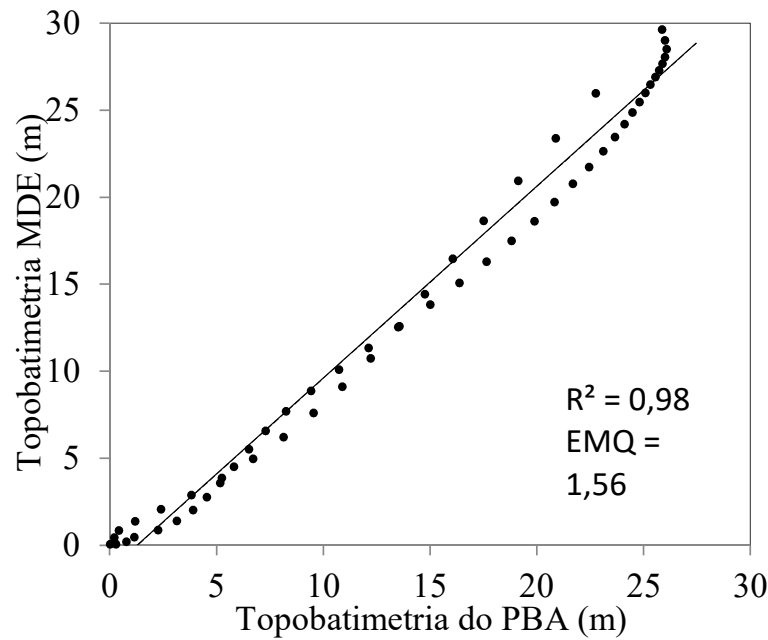

Figura 6. Gráfico de dispersão da seção topobatrimétrica constatada no PBA e extraída do MDE.

Figure 2. Scatterplot of the cross section verified in the Basic Environmental Project and extracted from the DEM.

\section{DISCUSSÃO}

A estimativa das alturas de barragem com base no MDE disponibilizado gratuitamente pelo TOPODATA dispensa recursos humanos quando comparado, por exemplo, com a pesquisa de campo em projetos ou processos ambientais que contenham essa informação. $\mathrm{O}$ fato de esta informação estar inserida no ambiente SIG possibilita estudos mais complexos como comportamento hidráulico, sedimentologia, contenção de cheias.
$\mathrm{O}$ erro absoluto das alturas estimadas, frente às de projeto, foi igual ou inferior a 5 metros, menor que os 6 metros, constatado por Rabus et al. (2003) e Rexer; Hirt, (2014), ao analisar a precisão altimétrica dos dados do SRTM (base de dados do TOPODATA). Rabus et al. (2003) mencionam essa precisão vertical, relativa, a escala local de $200 \mathrm{~km}$. Considerando ainda que a escala utilizada nesse estudo foi de $1 \mathrm{~km}$.

Ainda que a seção extraída do MDE possua precisão de 30 metros, menor que a do projeto, com pontos de controle de 10 metros, houve notável, precisão da seção extraída do MDE com relação à de projeto. $\mathrm{O}$ erro médio quadrático calculado entre os dados altimétricos (das secções) foi de 1,56 metros, menor que os 3,64 metros, calculado por Marini et al. (2017), em estudos de validação de precisão altimétrica do MDE TOPODATA.

\section{CONCLUSÕES}

As duas maneiras de analisar a precisão do MDE, com base na altura da barragem e secção topobatimétrica, favorece a aplicabilidade de métodos de validação, por conta da maior facilidade em obter informação de altura de barragem, por exemplo.

A análise de precisão evidenciou que há erros em relação às informações extraídas do MDE, mas considerando a escala utilizada nesse estudo, os erros foram menores que os observados na literatura. Vale observar que a barragens analisadas são relativamente altas (com no mínimo de 12 metros de altura). A validação do MDE com base em barragens menores pode gerar erros consideráveis.

Diversos fatores influenciam a precisão do MDE, incluindo: a fonte dos dados, o algoritmo usado para calcular diferentes atributos, elevação e resolução espacial (LI et al., 2017). É preciso considerar, também, a necessidade de diversos estudos de validação, seja eles locais e/ou considerando bases de dados diferentes. Para assim determinar as limitações e aplicações do MDE.

\section{REFERENNCIAS}

DIXON, B.; EARLS, J., Resample or not?! Effects of resolution of DEMs in watershed modeling. Hydrological processes, Chichester, v. 23, n. 12, p. 1714-1724, apr. 2009. DOI: http://dx.doi.org/10.1002/hyp.7306

ERSKINE, R. H.; GREEN, T.R.; RAMIREZ, J. A.; MACDONALD, L. H. Digital Elevation Accuracy and Grid Cell Size: Effects on Estimated Terrain Attributes. Soil Science Society of America Journal, Madison, v. 71, n. 4, p. 1371-1380, may. 2007. DOI: http://dx.doi.org/10.2136/sssaj2005.0142

GHUMMAN, A. R.; AL-SALAMAH, I.S.; ALSALEEM, S. S.; HAIDER, H. Evaluating the impact of lower resolutions of digital elevation model on rainfall-runoff modeling for ungauged catchments. Environmental Monitoring and Assessment, v. 189, n. 54, jan. 2017. DOI: https://dx.doi.org/10.1007/s10661-017-5766-0

GONGA-SAHOLIARILIVA, N.; GUNNELL, Y.; PETIT, C.; MERING, C. Techniques for quantifying the accuracy of gridded elevation models and for mapping uncertainty in digital terrain analysis. Progress in Physical Geography, London, v. 35, n. 6, p. 739-764, jul. 2011. DOI: https://dx.doi.org/10.1177/0309133311409086 
HABTEZION, N.; TAHMASEBI NASAB, M.; CHU, X. How does DEM resolution affect microtopographic characteristics, hydrologic connectivity, and modelling of hydrologic processes?. Hydrological processes, Chichester, v. 30, n. 25, p. 4870-4892, dez. 2016. DOI: http://dx.doi.org/10.1002/hyp.10967

LARENTIS, D. G.; COLLISCHONN, W.; OLIVEIRA F.; TUCCI C. E. M. Gis-based procedures for hydropower potential spotting. Energy, Oxford, v. 35, n. 10, p. 4237 4243, out. 2010.2 DOI: https://dx.doi.org/10.1016/j.energy.2010.07.014

LI, X.; ZHANG, Y.; JIN, X.; HE. Q.; ZHANG, X. Comparison of digital elevation models and relevant derived attributes. Journal of Applied Remote Sensing, Bellingham, v. 11, n. 4, p. 1-23. dez. 2017. DOI: https://dx.doi.org/10.1117/1.JRS.11.046027

LIN, S.; JING, C.; COLES, N. A.; CHAPLOT, V.; MOORE, N. J.; WU, J.; Evaluating DEM source and resolution uncertainties in the soil and water assessment Tool. Stochastic Environmental Research and Risk Assessment, Berlin, v. 27, n. 1, p. 209-221, 2013. DOI: https://dx.doi.org/10.1007/s00477-012-0577-x

LÓPEZ-VICENTE, M.; ÁLVAREZ, S. Influence of DEM resolution on modelling hydrological connectivity in a complex agricultural catchment with woody crops. Earth Surface Processes and Landforms, Sussex, v. 43, n. 7, p. 1403-1415, jan. $2018 . \quad$ DOI: http://dx.doi.org/10.1002/esp.4321

MARINI L. B.; JUNIOR, J. M.; RAMOS, A. P. M.; PARANHOS FILHO, A. C.; BARROS, W. M.; HIGA, L. Digital Surface Models and their Application to the 3D Representation of Nhecolândia Pantanal. Anuário do Instituto de Geociências, Rio de Janeiro, v. 40, n. 3, p. 4854, set. $2017 . \quad$ DOI: http://dx.doi.org/10.11137/2017_3_48_54

RABUS, B.; EINEDER, M.; ROTH, A.; BAMLER, R. The shuttle radar topography mission- a new class of digital elevation models acquired by spaceborne radar. ISPRS Journal of Photogrammetry and Remote Sensing, Amsterdam, v. 57, n. 4, p. 241-262, fev. 2003. DOI: https://dx.doi.org/10.1016/S0924-2716(02)00124-7

REDDY, A. S.; REDDY, M. J. Evaluating the influence of spatial resolutions of DEM on watershed runoff and sediment yield using SWAT. Jornal of Earth System Science, New Delhi, v. 124, n. 7, p. 1517-1529, set. 2015. DOI: https://dx.doi.org/10.1007/s12040-015-0617-2

REXER, M.; HIRT, C. Comparison of free high resolution digital elevation data sets (ASTER GDEM2, SRTM v2.1/v4.1) and validation against accurate heights from the Australian national gravity database. Australian Journal of Earth Sciences, Victoria, v. 61, n. 2, p. 213-226, out. 2014.

DOI: https://dx.doi.org/10.1080/08120099.2014.884983

SOUSA, T. M. I.; PAZ, A. R. How to evaluate the quality of coarse-resolution DEM-derived drainage networks. Hydrological Processes. Chichester, v. 31, p. 3379-3395, ago. 2017. DOI: https://dx.doi.org/10.1002/hyp.11262

TAN, M. L.; FICKLIN, D. L.; DIXON, B.; YUSOP, Z.; CHAPLOT, V. Impacts of DEM resolution, source, and resampling technique on SWAT-simulated streamflow. Applied Geography, Oxford, v. 63, p. 357-368, set. 2015. DOI: https://dx.doi.org/10.1016/j.apgeog.2015.07.014

WALKER, J. P.; WILLGOOSE, G. R. On the effect of digital elevation model accuracy on hydrology and geomorphology. Water Resources Research, Washington, v. 35, n. 7, p. 2259-2268, jun. 1999. DOI: http://dx.doi.org/10.1029/1999WR900034

ZHOU, L.; XIONG. L. Natural topographic controls on the spatial distribution of poverty-stricken counties in China, Applied Geography, Oxford, v. 90, p. 282-292, jan. 2018. DOI: https://dx.doi.org/10.1016/j.apgeog.2017.10.006 